\title{
NUMERICAL SIMULATION OF STEAM CONDENSATION IN A NOZZLE
}

\author{
Jan HALAMA* and Jaroslav FOŘT ${ }^{*}$ \\ Abstract: This paper describes a model of compressible flow including homoge- \\ nous nucleation and consequent growth and evaporation of droplets. Considered \\ fluid is water vapor with relatively small mass fraction of dispersed water droplets. \\ Main issues of numerical simulation based on this model are discussed. Results \\ of numerical simulation are compared with experimental data.
}

\section{INTRODUCTION}

Steam in a typical technical application expands and thus usually moves from dry (one phase) into wet (two-phase) region. The appearance of a liquid phase is interesting from many points like thermodynamic efficiency, flow unsteadiness, surface erosion etc. Presented flow model relies on classical nucleation theory of Becker and Doering [1], which is complemented by certain empirical correction. We use the correction for surface tension proposed by Petr and Kolovratnik [2]. Droplets dispersed in elemental volume (from the point of view of used domain discretization - detail are explained in the section about method of solution) have different sizes. There exists different methods how to describe the size spectra e.g. distribution function, discrete size classes, etc. We use the method of moments according to Hill [3], where the whole size spectra is described by a few integral parameters called moments and all droplets in elemental volume are represented by average size. This assumption works well for simple problems like flow in a nozzle, where all droplets appear at once in a small region, therefore they have similar sizes and then they grow or evaporate in a similar way. This model can be quite successfully used also for flow in a single turbine cascade. Accuracy problems of such model can appear in the case of secondary nucleation, i.e. if the mixture of vapor with dispersed droplets is subjected to rapid expansion, which results in further nucleation. The mixture then contains two (or more) groups of droplets with different sizes and the Hill's model gives the average size somewhere between. It has influence on droplet growth speed, since it depends on droplet size. The situation is even more complicated for flow simulation in a multistage turbine, where nucleation can appear repeatedly.

\section{MOdEL OF CONDENSING STEAM FLOW}

Assume two-dimensional flow of mixture of water vapor with dispersed water droplets treated as a continuum. We consider a modification of model originally used by Šejna [4]. The model consists of 2D Euler equations with additional transport equations for mass fraction of liquid phase $\chi$ (wetness) and integral parameters (moments) $Q_{0}, Q_{1}$ and $Q_{2}$

$$
Q_{0}=N, \quad Q_{1}=\sum_{i=1}^{N} r_{i}, \quad Q_{2}=\sum_{i=1}^{N} r_{i}^{2},
$$

where $N$ is the total number of droplets per unit mass and $r_{i}$ is the radius of $i$-th droplet. The system of equations reads

$$
\partial_{t} \mathbf{W}+\partial_{x} \mathbf{F}+\partial_{y} \mathbf{G}=\mathbf{Q},
$$

\footnotetext{
* Department of Technical Mathematics, Faculty of Mechanical Engineering, Czech Technical University Prague, Karlovo nám. 13, CZ-12135 Praha 2, Czech Republic, Jan.Halama@fs.cvut.cz, Jaroslav.Fort@fs.cvut.cz
}

This is an Open Access article distributed under the terms of the Creative Commons Attribution License 2.0, which permits unrestricted use, distribution, and reproduction in any medium, provided the original work is properly cited. 


$$
\frac{\partial}{\partial t}\left[\begin{array}{c}
\rho \\
\rho u \\
\rho v \\
e \\
\rho \chi \\
\rho Q_{2} \\
\rho Q_{1} \\
\rho Q_{0}
\end{array}\right]+\frac{\partial}{\partial x}\left[\begin{array}{c}
\rho u \\
\rho u v \\
\rho \chi u \\
\rho Q_{2} u \\
\rho Q_{1} u \\
\rho Q_{0} u
\end{array}\right]+\frac{\partial}{\partial y}\left[\begin{array}{c}
\rho v \\
\rho v u \\
\rho v^{2}+p \\
(e+p) v \\
\rho \chi v \\
\rho Q_{2} v \\
\rho Q_{1} v \\
\rho Q_{0} v
\end{array}\right]=\left[\begin{array}{c}
0 \\
\frac{4}{3} \pi r_{c}^{3} \rho_{l} J+4 \pi \rho Q_{2} \dot{r} \rho_{l} \\
r_{c}^{2} J+2 \rho Q_{1} \dot{r} \\
r_{c} J+\rho Q_{0} \dot{r} \\
J
\end{array}\right] .
$$

This system is closed by the equation for pressure $p$ (the same value for both phases)

$$
p=\frac{(\gamma-1)(1-\chi)}{1+\chi(\gamma-1)}\left[e-\frac{1}{2} \rho\left(u^{2}+v^{2}\right)+\rho \chi L\right] .
$$

Symbol $\rho$ refers mixture density, $u$ and $v$ mixture velocity components, $e$ total mixture energy. The specific heat ratio $\gamma=\gamma(T)$ is taken as a function of temperature $T$ as well as $L=L(T)$ the latent heat of condensation-evaporation change. The model is valid also for dry steam, i.e. $\chi=Q_{0}=Q_{1}=Q_{2}=0$. Condensation of vapor in nozzles and turbines starts usually when the vapor temperature falls significantly below the saturation temperature due to high speed of expansion. The model of nucleation proposed by Becker and Döring [1] takes into account such non-equilibrium behavior. The number of new droplets per second and per unit volume is defined as

$$
J=\sqrt{\frac{2 \sigma}{\pi m_{v}^{3}}} \cdot \frac{\rho_{v}^{2}}{\rho_{l}} \cdot \exp \left(-\beta \cdot \frac{4 \pi r_{c}^{2} \sigma}{3 k_{B} T_{v}}\right) .
$$

The surface tension $\sigma=\sigma(T)$ is corrected by the parameter $\beta$ according to Petr and Kolovratník [2] to match correctly the start of nucleation. The Figures 1 and 2 show the effect of this correction on pressure distribution and wetness respectively for the flow in the Barschdorf nozzle (more information about Barschdorf nozzle problem see in the section ). Note that numerical method for the case with

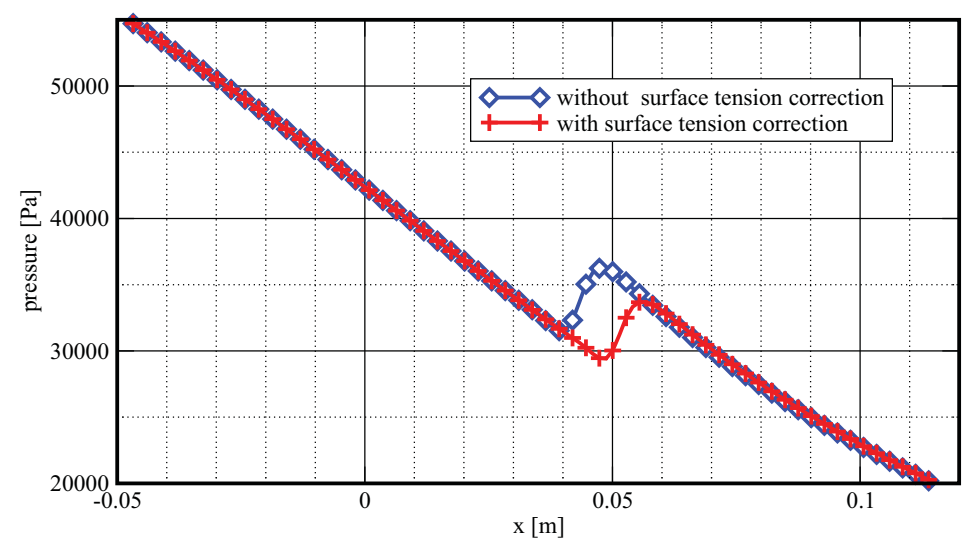

Figure 1: Pressure distribution along the Barschdorff nozzle axis for $T_{01}=380.50 \mathrm{~K}$.

$T_{01}=373.15 K$ yields unsteady oscillating solution, if no correction is used opposite to the steady solution, when the correction is applied. The size of new droplets is given by critical radius

$$
r_{c}=\frac{2 \sigma}{L \rho_{l} l n\left(T_{s} / T\right)} .
$$




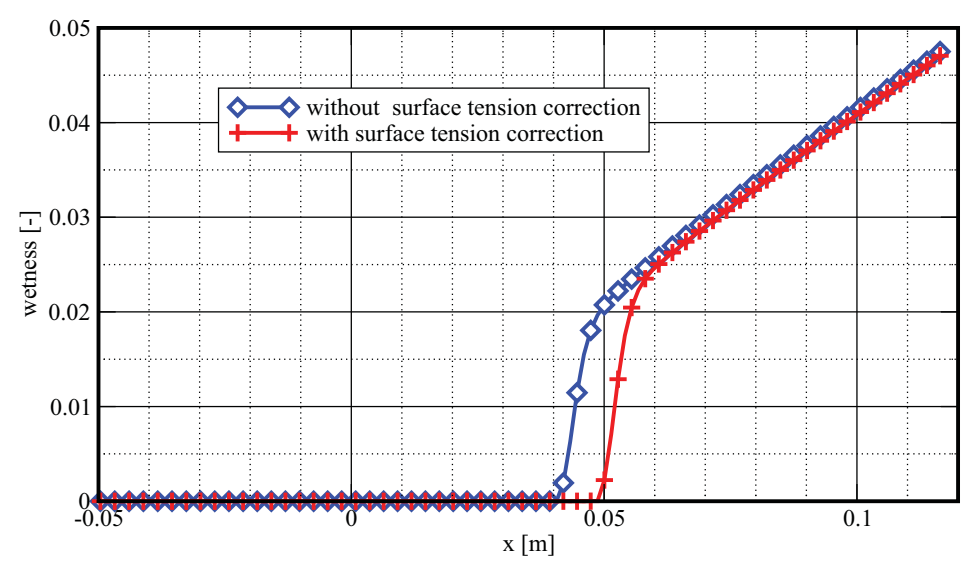

Figure 2: Wetness along the Barschdorff nozzle axis for $T_{01}=380.50 \mathrm{~K}$.

The droplet growth speed is given by

$$
\dot{r}=\frac{\lambda_{v}\left(T_{s}-T\right)}{L \rho_{l}(1+3.18 \cdot K n)} \cdot \frac{r-r_{c}}{r^{2}},
$$

where the saturation temperature $T_{s}$ is computed by the library function of IAPWS-IF97. Average droplet radius $r$ is taken as

$$
r=\sqrt{Q_{2} / Q_{0}} .
$$

\section{Method OF SOlution}

The current implementation of finite volume method uses domain of solution (nozzle or one blade chanel of cascade) discretized by the body fitted quadrilateral structured mesh, which is generated before computation. The mesh is refined in regions where nucleation is expected. The problem is solved like evolution problem with chosen initial conditions and time independent boundary conditions - the so called time marching method. This method can be used to obtain steady as well as unsteady (time accurate method has to be used) solution. The flow model takes into account phenomena like convection, nucleation and droplet growth or evaporation. These phenomena have unfortunately very different time scales, especially nucleation is very fast. Therefore used numerical method is based on the so called fractional step method of Strang [5], where each step of time evolution is divided into sub-steps where convection and condensation are solved separately by different methods (Lax-Wendroff finite volume method for convection and Runge-Kutta method for condensation).

The flow model has to be implemented into numerical method in a careful way. For example one has to avoid the division of two small numbers in the Eq. (8) for small values of wetness. Also the Eq. (7) for the droplet growth speed contains critical radius computed by the Eq. (6), which goes to infinity, if the vapor temperature approaches saturation temperature. This growth is compensated by the term $T_{s}-T$ in the Eq. (7). It leads to the undesired term of type 'zero multiplied by infinity', we use the Taylor expansion of $\dot{r}$ in $T=T_{s}$ to avoid such term.

The following realization of boundary conditions is used for presented nozzle flow problems: at the inlet (we expect subsonic inlet flow velocity) the Mach number is evaluated along the inlet boundary from values taken from inside of solution domain. This Mach number and given values (from outside of solution domain) of $p_{01}, T_{01}, \chi, Q_{2}, Q_{1}$ a $Q_{0}$, flow direction and isentropic flow relations are used to update values of vector of unknowns. At the outlet boundary we use 'do nothing' type of boundary conditions (we expect supersonic flow velocity there).

\section{COMPARISON WITH EXPERIMENTAL DATA}

The applicability of condensation model and proposed method of solution has been checked for the flow in a convergent-divergent nozzle. We consider dry steam at the nozzle inlet and nucleation starting in a divergent part of a nozzle. The flow along the nozzle axis is mainly driven by potential effects, i.e. it can be approximated by inviscid flow model. Also the Hill's method should be enough precise in that case. Two different flow regimes have the same value of inlet total pressure $p_{01}=78390 \mathrm{~Pa}$ and different 
total inlet temperatures. Nucleation releases heat in a relative narrow region, therefore a pressure jump called the condensation shock appears. The figure 3 shows a well captured position of this shock. The figure 4 shows the comparison of results of presented method with the numerical results of S. Dykas [7].

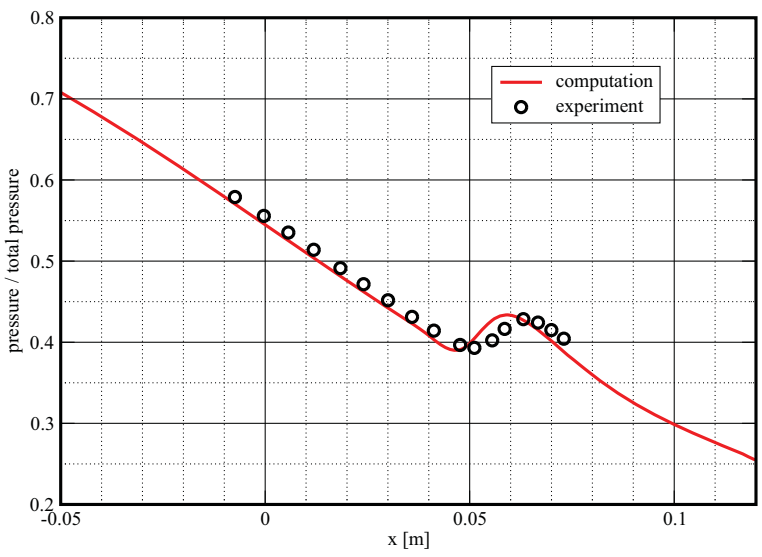

(a) $T_{01}=380.50 K$

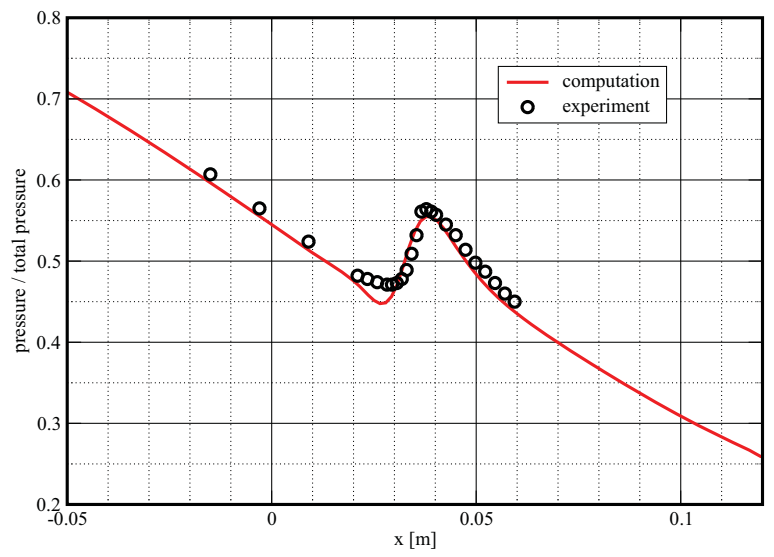

(a) $T_{01}=373.15 K$

Figure 3: Pressure distribution along the nozzle axis, experiment [6].
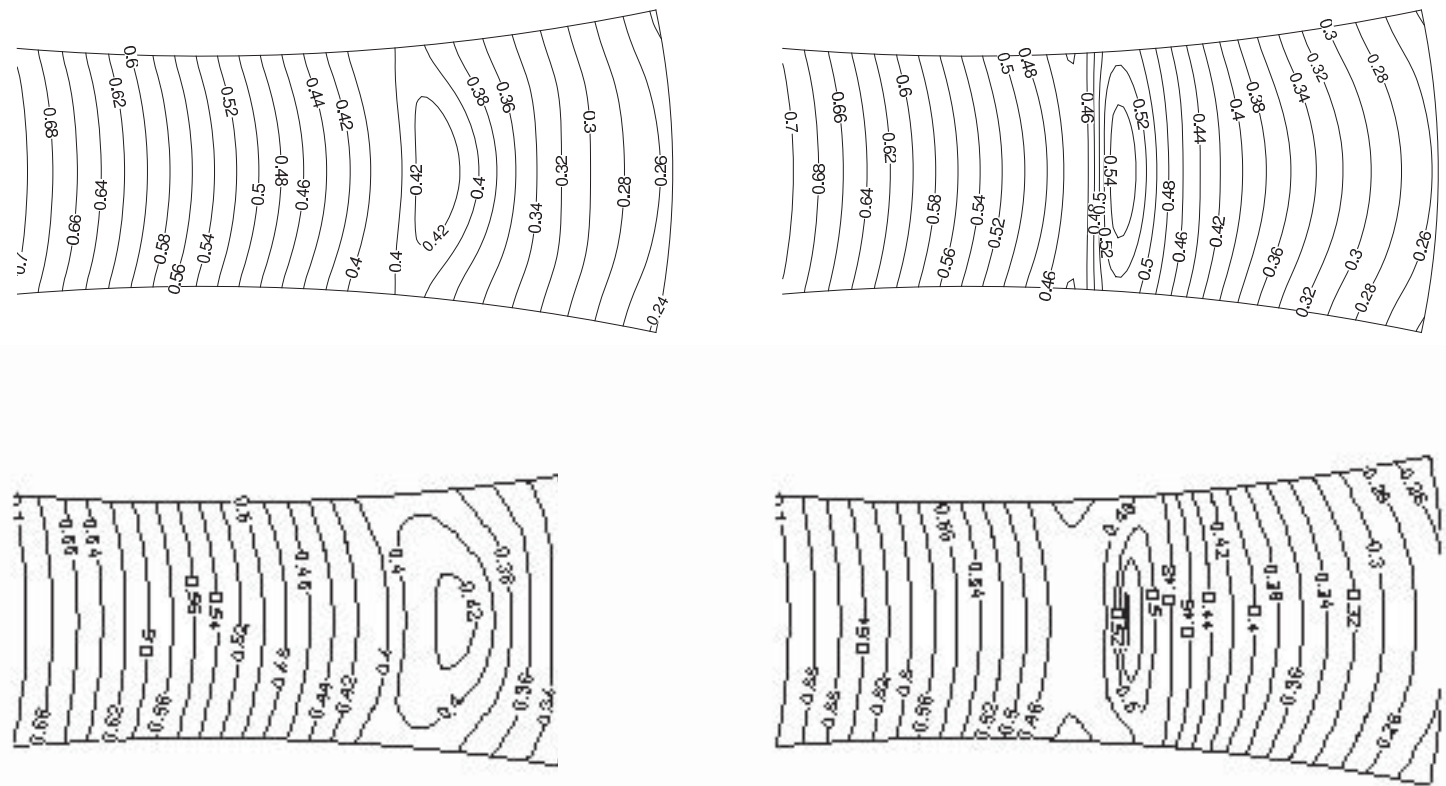

Figure 4: Isolines of pressure $p / p_{0}\left(\Delta p / p_{0}=0.02\right)$ for the Barschdorff nozzle, $T_{01}=380.50 K$ (on the left), $T_{01}=373.15 \mathrm{~K}$ (on the right), results of presented numerical method in upper row and results of S. Dykas [7] in lower row.

\section{CONCLUSIONS}

Presented method for computation of condensing steam flow yields a good results in the case of nozzle flow (continuous expansion). Careful implementation of the model provides a sufficient robustness also for computations of flow in turbine cascades. Next we plan to improve parts of flow model: equation of state and the droplet growth speed computation. 


\section{REFERENCES}

[1] Becker R., Döring W.: Kinetische Behandlung der Keimbildung in übersättingten Dämpfen, Journal Ann. d. Physik, Vol. 24, No. 8, 1935.

[2] Petr V., Kolovratník M.: Heterogenous Effects in the Droplet Nucleation Process in LP Steam Turbines, 4th European Conference on Turbomachinery, Firenze, 2001

[3] Hill P.G.: Condensation of water vapor during supersonic expansion in nozzles, part 3, Journal of Fluid Mechanics, Vol.3, 1966, 593-620.

[4] Šejna M.: Numerical modeling of two-phase flow of steam with homogenous condensation, Doctoral thesis, CTU Prague 1995, (in czech).

[5] Strang G.: On the construction and comparison of difference schemes, SIAM Journal of Numerical Analysis, Vol. 5, 1968, 506-517.

[6] Barschdorff D.: Verlauf der Zustandgroesen und gasdynamische Zuammenhaenge der spontanen Kondensation reinen Wasserdampfes in Lavalduesen, Forsch. Ing.-Wes., Vol. 37, No. 5, 1971, (in german).

[7] Dykas S., Goodheart K., Schnerr G.H.: Numerical study of accurate and efficient modeling for simulation of condensing flow in transonic steam turbines, 5th European conference on Turbomachinery, Prague 2003, 751-760.

\section{ACKNOWLEDGMENTS}

The support of the Research plan MSM CR no. 6840770003 and the grant no. 101/11/1593 of GACR is greatly acknowledged. 